\title{
Interaction of turbulence with magnetic islands: effect on bootstrap current.
}

\author{
W.A. Hornsby ${ }^{1} \ddagger$, M. Siccinio ${ }^{2}$, A.G. Peeters ${ }^{3}$, E. Poli ${ }^{2}$, \\ A.P. Snodin ${ }^{1}$, F.J. Casson ${ }^{1}$, Y. Camenen ${ }^{1}$, G. Szepesi ${ }^{1}$ \\ ${ }^{1}$ Centre for Fusion, Space and Astrophysics, Dept. of Physics, University of Warwick, \\ Coventry, U.K. \\ E-mail: William.Hornsby@uni-bayreuth.de \\ ${ }^{2}$ Max-Planck-Institut für Plasmaphysik, Boltzmannstrasse 2, D-85748 Garching bei \\ München, Germany \\ ${ }^{3}$ Universität Bayreuth, Bayreuth, Germany
}

\begin{abstract}
.
A finite radial temperature gradient has been observed to be maintained within magnetic islands in gyro-kinetic turbulence simulations despite the fast motion along the field, and is related to the trapped particles which do not move along the field around the island. Recent calculations of the interaction of drift wave turbulence with magnetic islands have shown that turbulence can exist within the separatrix, which in turn allows only the partial flattening of electron temperature profiles. Here we calculate, using a minimal drift-kinetic model, the effect on the bootstrap current in a tokamak. Consequences for the stability of the neo-classical tearing mode are discussed.
\end{abstract}

\section{Introduction}

The tearing mode is a resistive magnetohydrodynamic instability that, by the process of reconnection, perturbs the confining magnetic field to introduce magnetic island structures. The parallel streaming along the perturbed field lines, causes a flattening of the radial density and temperature profiles through the island, leading to a detrimental effect on confinement.

The stability of magnetic islands generated by the tearing mode is determined by the current around the rational surface on which the island is situated[1].

Contributions to the current density come from various sources, including the induced current[2], bootstrap current [3, 4] and polarisation current[5]. Furthermore, it is thought that turbulence will have a significant effect on the stability of the mode[6, 7,8$]$. The current profile can be modified by the presence of the island itself and flattening of density and temperature profiles in island vicinity.

$\ddagger$ Present address: Universität Bayreuth, Bayreuth, Germany 
In this paper we will concentrate on the bootstrap current drive to the tearing mode. This leads to a modification of the Tearing Mode, known as the Neoclassical Tearing Mode (NTM) $[3,4]$.

The bootstrap current is produced by the plasma itself and flows in the direction of the magnetic field lines. Background density and temperature gradients create an asymmetry in the trapped population which is transferred on to the passing population by their collisional interaction. It is this asymmetry, and a further asymmetry between the electrons and ions due to their charge, which forms the bootstrap current [9]. A simple model for the bootstrap current is to state that it is directly proportional to the radial pressure gradient.

The presence of a magnetic island helps to flatten the radial pressure profiles as it acts as a short circuit between flux surfaces. This effect removes the drive for the bootstrap current within the island[10]. It is this perturbation in the bootstrap current, caused by the island itself, that can drive the tearing mode unstable and cause the island to grow. This growth can have a catastrophic effect on the confinement as the radial component of the magnetic field that the island introduces can greatly enhance the radial heat and particle flow.

Previous work using gyro-kinetic simulations has found that the temperature gradients through the island O-points are further modified by the action of drift-wave turbulence around and within the island[11, 12, 14, 13]. These calculations, however, did not allow the direct calculation of the bootstrap current to determine its full effect. To address this point, presented here is a model problem in one spatial and velocity coordinate, that contains all the necessary physics ingredients to enable the calculation of the effect of turbulence on the bootstrap current, and thus allows us to further comment on the stability of the NTM.

This paper is organised as follows. The first section (Section 2) will summarise the results of three-dimensional nonlinear gyro-kinetic simulations of turbulence in and around coherent magnetic island structures. In the second part (Sections 3 and 4) we will outline a simple $1 \mathrm{D}-1 \mathrm{~V}$ model used to calculate the effect of turbulence on the temperature profiles, and in turn, the bootstrap current profile through the island.

\section{Effect of Magnetic islands on drift-wave turbulence.}

Recent advances in computational power have enabled the first truly multi-scale selfconsistent simulations of turbulence to be performed[12, 14, 15]. Gyro-kinetic and fluid simulations[16, 17, 18, 13, 19] of the influence of large magnetic islands on driftwave instabilities and micro-scale turbulence have uncovered new physics. Furthermore recent analytic work has provided fresh insights into the multi-scale interaction of microinstabilities and large scale magnetic islands[20, 8, 21].

The presence of a magnetic island flattens the pressure profiles through the island , which in turn removes the drive for the turbulence. As such electrostatic turbulence is mainly suppressed within the the island. Furthermore the presence of the island 


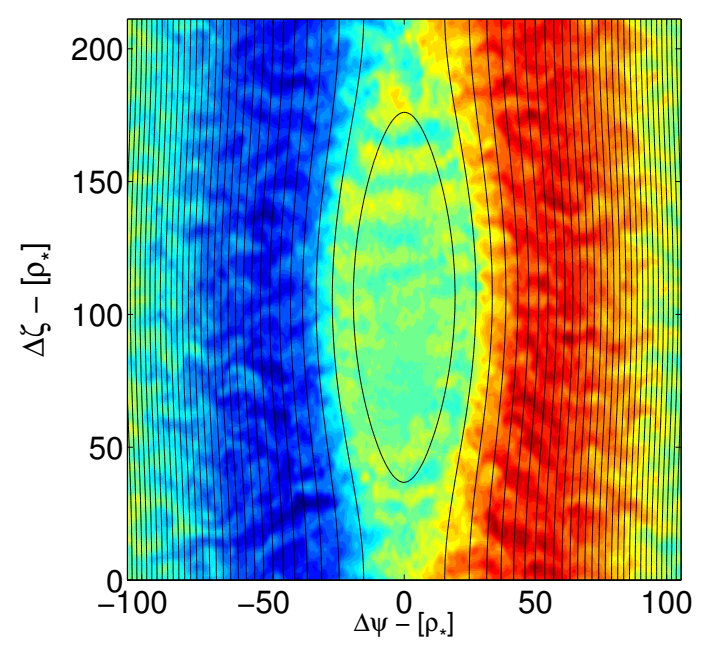

Figure 1. Normalized electrostatic potential $\left(\phi_{N}=e \phi / T \rho_{*}, w / \rho_{i}=24\right)$ in the plane perpendicular to the magnetic field (outboard midplane). Black lines represent the perturbed flux surfaces calculated from the total vector potential. The presence of the island embedded in the turbulence not only generates flows around the island structure. It is also seen that turbulence, which is not driven within the island, can enter the island via spreading.

modifies the generation of zonal flows, generated by the turbulence. These flows now move around the island and help advect heat radially. A further flow around the island can be generated by a nonlinearly driven potential vortex that forms within the island depending on the scale separation between the island mode and the turbulent modes. The combined effect of these flows is that the presence of a large magnetic island is detrimental to the heat confinement of a Tokamak.

The work of Ref.[12] has revealed that the radial electron temperature gradient is resilient to the presence of the island. Closer inspection of the particle dynamics shows that the trapped and passing electron populations have differing responses. The exact form of the temperature profiles through a magnetic island is determined through the competition between the transport parallel and perpendicular to the perturbed field lines[22]. This is encapsulated for the passing particles by the heat equation, $\chi_{\|} \nabla_{\|}^{2} T+\chi_{\perp} \nabla_{\perp}^{2} T=0$. Where $\chi_{\|}$and $\chi_{\perp}$ are the heat conduction coefficients parallel and perpendicular to the perturbed field lines respectively, and are assumed to be time and spatially constant. This equation neglects the effect of trapped particles, furthermore it assumes that there are no sources of sinks of heat in the vicinity of the island. The perpendicular transport in the heat equation is widely acknowledged to be due to gyroradius scale turbulence.

Here we perform a comparison of the transport time-scales. The parallel transport time-scale is determined by the time taken for a particle to traverse the island by motion along the field line. Consider an island based around a resonant surface $q=m / n$ where $\mathrm{m}$ and $\mathrm{n}$ are the mode numbers poloidally and toroidally respectively. The timescales 
for a particle to traverse the field around the island $\left(\tau_{s \|}\right.$, species denoted by letter s) by assuming that transport is advective and the field line has a length

$$
L_{\|}=\frac{4 \pi q R}{m w \hat{s}} .
$$

can be estimated to be

$$
\frac{\tau_{s \|} v_{t h i}}{R}=\sqrt{\frac{T_{i} m_{s}}{T_{s} m_{i}}} \frac{4 \pi q}{\left(k_{\theta}^{I} \rho\right) \hat{s}(w / \rho)} .
$$

where $k_{\theta}^{I}$ is the wavenumber of the island mode. $\hat{s}$ is the magnetic shear and $\mathrm{q}(\mathrm{q}=\mathrm{m} / \mathrm{n})$ is the safety factor and $\mathrm{R}$ is the major radius of the torus.

The timescale for perpendicular transport across the island $\tau_{s \perp}$ is assumed to be diffusive with the length scale of the island half width, w,

$$
\frac{\tau_{s \perp} v_{t h i}}{R}=\frac{1}{2 \chi_{s}^{N}}\left(\frac{w}{\rho_{i}}\right)^{2}
$$

where $\chi_{s}^{N}$ is the relevant transport coefficient in gyro-Bohm units $\left(\chi_{s}=\chi_{s}^{N} \rho_{i}^{2} v_{t h i} / R\right)$, $\mathrm{R}$ is the major radius and $v_{t h i}$ being the ion thermal velocity.

For the parameters used in the simulation shown, the mode wavenumber is $\left(k_{\theta}^{I} \rho_{i}=0.025\right)$, the parallel ion transport timescale is $\tau_{i \|} \approx 196$, the electron parallel transport time scale is $\tau_{e \|} \approx 3.3$, while the perpendicular timescales for the ions and electrons are $\tau_{i \perp} \approx 40$, and $\tau_{e \perp}=80$ (times are given in of $R / v_{t h i}$ ) respectively. The normalised density and temperature length scales are $R / L_{n e}=R / L_{n i}=2.2$ and $R / L_{T e}=R / L_{T i}=6.9$. From the comparison of the timescales one would expect a moderate flattening for the ions (quantified by the ratio $\tau_{\perp} / \tau_{\|}=5.0$ ) and a complete flattening of the electron temperature profile $\left(\tau_{\perp} / \tau_{\|}=24.2\right)$.

It is generally accepted that the fast transport along the magnetic field flattens the radial pressure gradient in the island, provided the perpendicular transport across the island is negligible against the parallel motion around the island [22] as encapsulated by the heat equation. For the timescales of our simulation we have previously estimated the electrons to have a zero temperature gradient inside the island due to their very rapid motion along the field line. The simulations, however, do not reproduce this effect as a finite electron temperature gradient is observed (See Fig. 2).

This observation has important consequences for the NTM. It is the gradient in the trapped population that is responsible for the bootstrap current (see Ref. [9] and the references cited therein). The rest of this paper will concern a minimal model of the electron dynamics around an island, to single out how the turbulence around the island affects the generation of the bootstrap current.

\section{Numerical model}

The essential ingredients to our minimal model are; the flattening of the pressure profiles due to the presence of the magnetic island, taking into account the different dynamics of the passing and trapped populations. We assume that the parallel transport time 


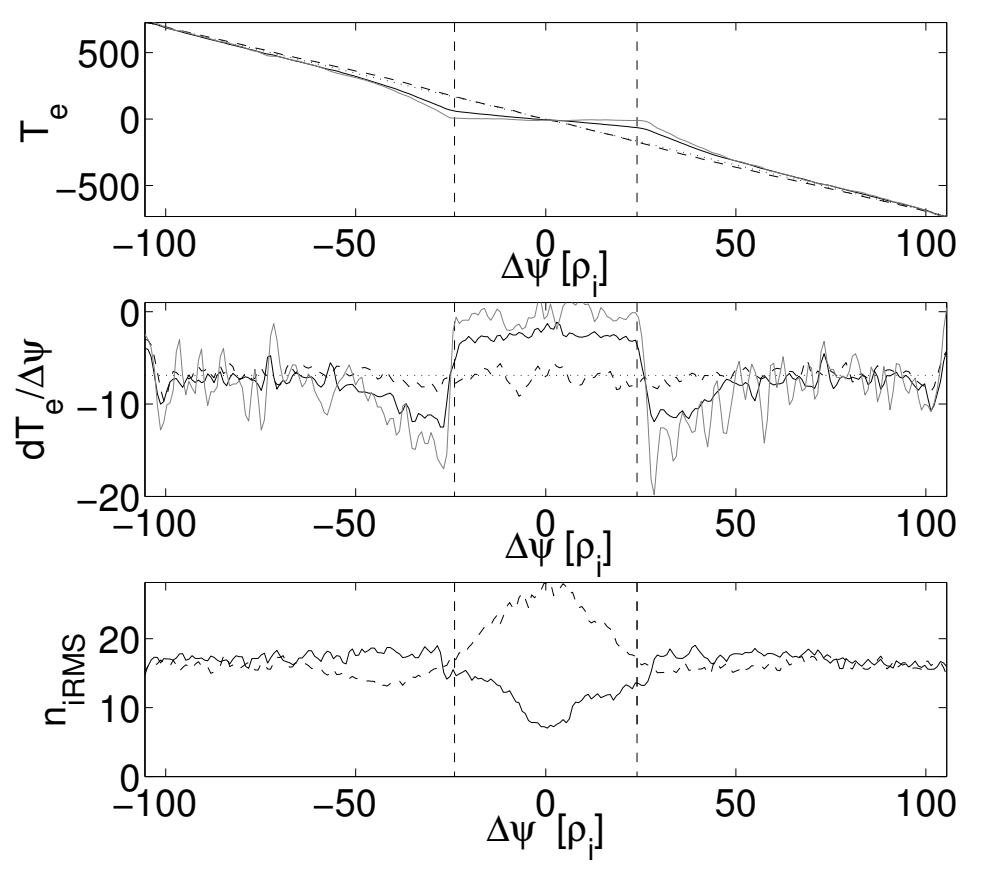

Figure 2. Top panel shows the radial electron temperature profiles, black: Low field side, gray: High field side. Middle panel shows the corresponding electron temperature gradients, black: Low field side, grey: High field side.. Bottom panel shows the RMS density fluctuation profile showing a decay of the fluctuation strength within the island. Dashed lines represent lines through the X-point in all three panels. (Reproduced with permission from Ref.[12].)

scale is significantly shorter than the perpendicular time scale for the passing particles. Specifically, looking at the limit where the ratio $\tau_{\perp} / \tau_{\|} \rightarrow \infty$ allows the study of the effect of the trapped particles only.

The diffusion of particles in velocity space due to collisions and, finally, the spreading and mixing of particles due to the presence of drift wave turbulence.

We consider first the bounce averaged equation for the distribution function $\langle f\rangle$ for the electrons as derived in $[23,25,24,26,27]$, where $\langle f\rangle$ is the perturbed distribution function along a banana trajectory. However since we propose a minimal model, we neglect the terms due to the effect of particle drifts apart from in the trapped population. We also only consider the radial direction at the outboard mid-plane of a Tokamak. So the kinetic equation for the bounce averaged distribution function becomes,

$$
\frac{d g}{d t}=C(g)
$$

Here the operator $d / d t$ is along a bouncing particle trajectory and $C(g)$ is the guiding centre collision operator.

The effect of drift-wave turbulence is modelled by a linear spatial diffusion operator,

$$
\frac{d\langle f\rangle}{d t}=D \frac{\partial\langle f\rangle}{\partial x^{2}}
$$



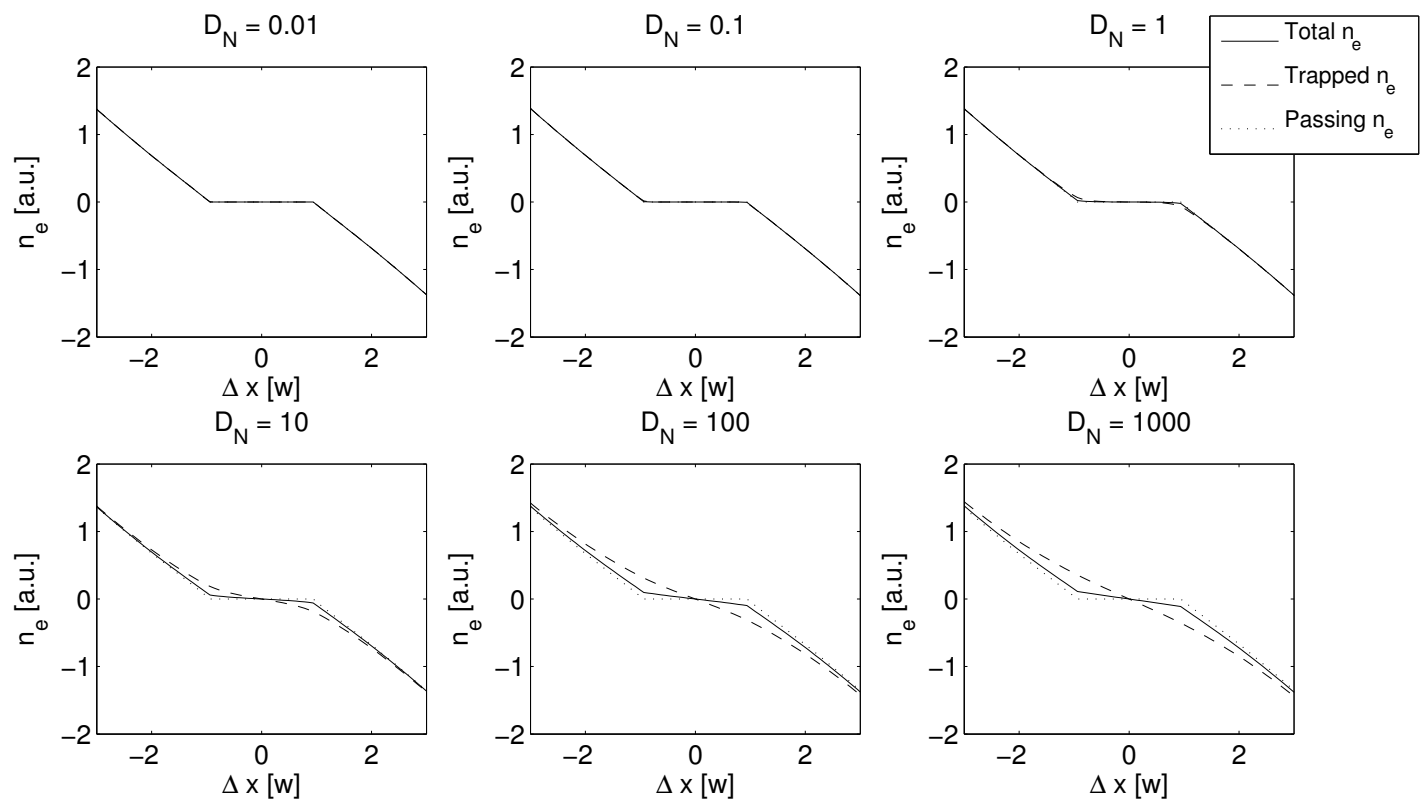

Figure 3. Plot of the temperature profiles as a function of the radial coordinate $x=r / w$ for various values of the normalised diffusion coefficient $D_{N}=D / w^{2} \nu_{e i}$. As the diffusion due to turbulence increases with relation to the collisionality, the effect on the trapped particle population becomes more pronounced.

The particles are assumed to have a single energy, roughly the thermal energy, and as such the distribution is only a function of the pitch angle $\lambda=\cos \theta$ (with $v_{\|}=v \cos \theta$ ).

In the equation above the radial coordinate is given by the bounce average of the particle position. This is the bounce point for the trapped particles. For passing particles all drifts will be neglected and the orbit lies inside a flux surface. These assumptions mean that the distribution function on the low field side of a flux surface $(x)$ is given by,

$$
g(x)=\langle f\rangle+\rho_{N} \lambda \frac{\partial\langle f\rangle}{\partial x},
$$

for trapped particles, and for passing particles

$$
g(x)=\langle f\rangle
$$

where

$$
\rho_{N}=\frac{m_{e} v}{e B_{\theta} w}
$$

is the poloidal gyro-radius normalised with the island width. We have assumed $\rho_{N}$ to be sufficiently small such that we can use the Taylor expansion in the equation above (in practice we have used $\rho_{N}=0.025$ below).

Consider a single particle energy, and as such the only collisions considered is pitch angle scattering. We evolve $\mathrm{g}$ using the Lorentz collision operator

$$
C(g)=\frac{\nu_{e i}}{2} \frac{\partial}{\partial \lambda}\left[\left(1-\lambda^{2}\right) \frac{\partial g}{\partial \lambda}\right]
$$


Where $\nu_{e i}$ is the electron-ion collision frequency which is the dominant collisional process. $\nu_{e i}$ is a function of velocity magnitude, $\mathrm{v}$, only and as such is constant in this model. This collision operator conserves both particle number and energy. Note that this acts on $\mathrm{g}$, the distribution function at the particle orbit, rather than $\mathrm{f}$, the bounce averaged function, and so to enact the collisions a transformation from $\mathrm{f}$ to $\mathrm{g}$ must occur.

The model described above includes all physics necessary for the description of trapped particle profile evolution through the island O-point and bootstrap current generation.

A finite radial gradient generates an asymmetry in the pitch angle distribution of the trapped particles and the bootstrap current is generated through the action of the collisions.

The island region $-w<x<w$, however demands special attention. The model presented is $1 \mathrm{D}-1 \mathrm{~V}$ and as such we need to make a modelling assumption to introduce the effect of flattening on the density and temperature profiles due to the parallel transport around the island. The fast parallel transport of electrons along the perturbed field lines is modelled by assuming that transport along the field lines is infinitely fast. Then:

$$
g_{\text {passing }}(-x)=g_{\text {passing }}(x)
$$

within the separatrix of the island $(-w<x<w)$. Technically this condition is enforced by averaging the passing distribution after each small time step. The consequence of this assumption is that the gradient in the passing distribution inside the island is always zero, consistent with what is seen for the passing population in the gyro-kinetic simulations.

Here time is normalised with the collision frequency $\nu_{e i}$ and the radial coordinate is normalised using the island width $x=\left(r-r_{s}\right) / w$, where $r_{s}$ is the radius of the resonant surface. (The island region is therefore $[-1,1]$ ). Consequently, the normalised diffusion coefficient is $D_{N}=D / w^{2} \nu_{e i}$, which is assumed to be constant.

The equations are solved on the x-domain $[-5,5]$ with boundary conditions $f=3$ at $x=-5$ and $f=-3$ at $x=5$. These boundary conditions help maintain a density gradient outside the island. While the condition above ensures that the trapped particle profile flattens within the island. A standard conservative second order central differencing is used.

\section{Results}

The results presented here were performed with 128 spatial grid points and 64 points in pitch angle, the trapped particles are represented by 10 velocity space points. The code is run until a steady state solution is maintained.

The distribution function for various values of the normalised diffusion coefficient is shown in Fig. 3. It can be seen that at sufficiently high normalised diffusion coefficient a finite gradient of trapped particles inside the island develops. The passing particle distribution is always flat as dictated by our model. 
This observation reinforces the statements made in the previous work, that the passing particles, which experience the full magnetic field perturbation flatten fully and are unaffected by the local turbulence, while the trapped particles are mixed by the turbulence which helps maintain a gradient in their population.

The gyro-kinetic simulations performed in ref. [12], due to the use of the collisionless approximation, describe the limit $D \rightarrow \infty$. It can also be seen that at sufficiently small diffusion coefficient the trapped particle distribution becomes flat. It is the collisional coupling to the passing distribution in combination with the long timescales for cross island diffusion that allows for this flattening.

This is in agreement with the statement made in the previous paper which was not directly based on a numerical simulation. With this simple model, this assertion has been verified.

In all of the simulations above the bootstrap current along the magnetic field line can be calculated through the integral

$$
J_{b s}=Q \int \mathrm{d} \lambda \lambda g(x, \lambda)
$$

Where $\mathrm{Q}$ is the charge of the species, which in this case is $Q=-e$. This current for various normalised diffusion coefficients is given in Fig. 4. Note that the current outside the island is not entirely constant due to the boundary conditions employed in the simulations. The averaged gradient across the box is pinned by the boundary conditions and a flattening inside the island leads to a steepening of the gradient outside the island and, therefore, a larger bootstrap current. The figure very clearly shows that in the case of small $D_{N}$, where collisions dominate over diffusion, the bootstrap current vanishes in the island. Whereas, in the case of high normalised diffusion coefficient, it remains finite within the separatrix, becoming less and less suppressed as the diffusion due to turbulence is increased.

The simulations using this model confirm the picture which was touched upon in [12], where by turbulence in and around a magnetic island suppresses the perturbation in the bootstrap current. In the weakly collisional case, the regime in which the gyrokinetic simulations were performed, a bootstrap current can remain inside the island which in turn eliminates the drive of the neoclassical tearing mode.

It is the gradient in the trapped population that is responsible for the bootstrap current. With the above observation we infer that the bootstrap current is not reduced inside the island and the drive for the NTM would be diminished in the presented case. While the density, the zero moment of the distribution function, in the passing particle population is flat through the island the collisional interaction with trapped particles helps maintain a gradient in the current, the first odd moment of the distribution function.

The neoclassical tearing mode is often observed to have a threshold behaviour, i.e. the mode only grows when the island size exceeds a threshold width[5]. What follows is an estimate for the critical width due to the turbulence.

De-trapping of electrons and ions, which occurs on a typical time scale $R \nu_{s} / r$, 

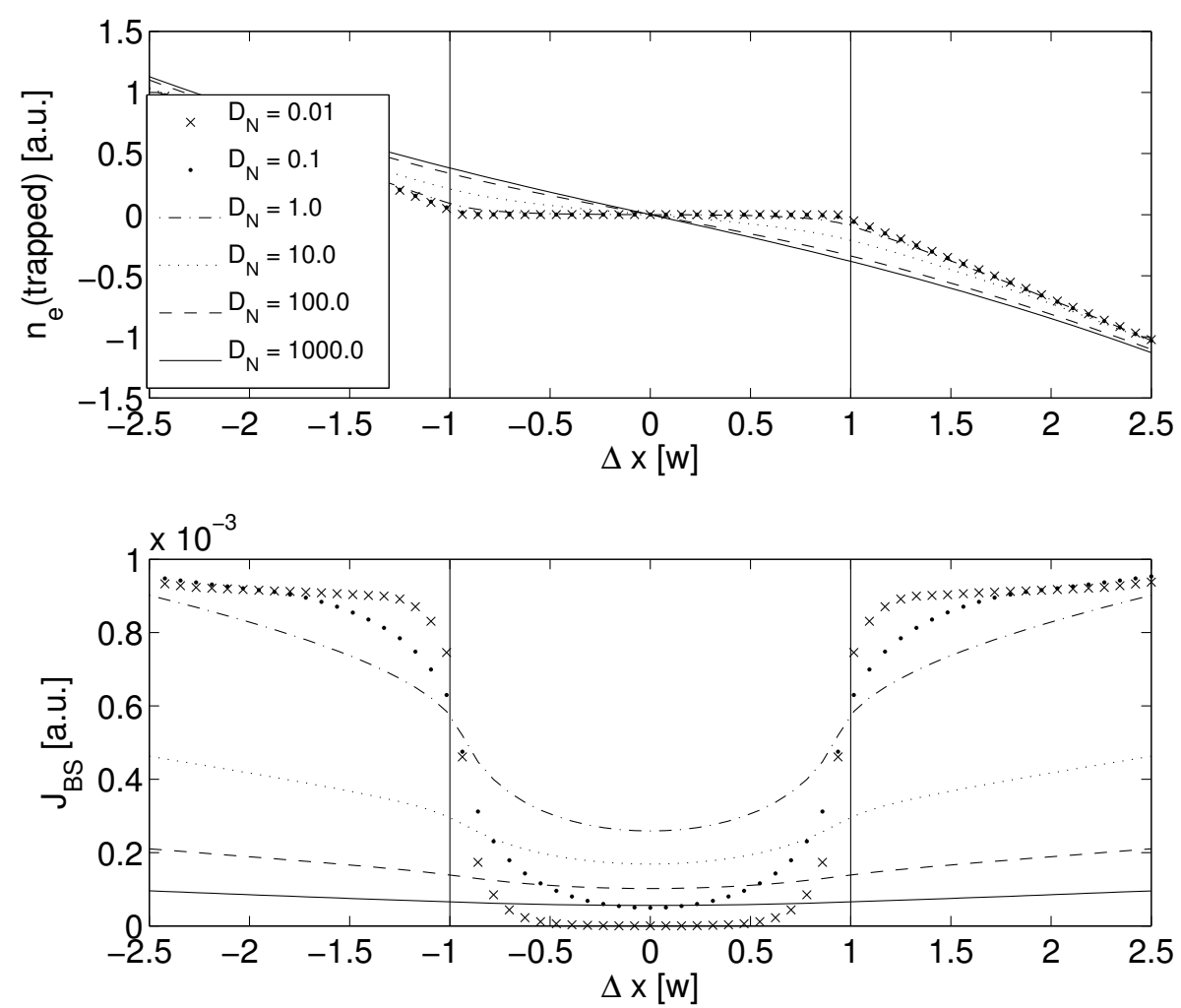

Figure 4. (top) The radial profile of the trapped electron population and (bottom) the bootstrap current profile in arbitrary units. Both are shown as a function of the normalised radius $x=r / w$ for various values of the normalised diffusion coefficient $D_{N}=D / w^{2} \nu_{e i}$. The island separatrix is denoted by the vertical grey line.

where $\nu_{s}$ is the collision frequency of species s, will reduce the temperature and density perturbation in the trapped region, provided it occurs on a timescale shorter than the perpendicular transport through the island.

Estimating the diffusion time for turbulence to spread across the island is,

$$
\tau_{D}=w^{2} / 2 D_{\perp} .
$$

while the electron trapping/de-trapping rate is given by:

$$
\tau_{T}=\epsilon / \nu_{e i} .
$$

Where $\epsilon=r / R$ is the inverse aspect ration. For the NTM drive to become effective, the timescales of the two processes must be comparable, which results in a critical island width

$$
w_{c}=\sqrt{2 D_{\perp} r / R \nu_{e i}}
$$

where $\mathrm{r}$ and $\mathrm{R}$ are the minor and major radii of a Tokamak respectively.

For a large Tokamak, such as ASDEX Upgrade, the following parameters $D=$ $3 \mathrm{~m}^{2} / \mathrm{s}, n_{e}=5.10^{19} \mathrm{~m}^{-3}$, and $T_{e}=3 \mathrm{keV}$, are used to estimate the seed island 
width. Used here is the calculated diffusion coefficient due to turbulence from Ref. [12] which, when normalised to gyro-Bohm units is $D_{N}=30$. The parameters used in this paper were derived from the cyclone base case[28], a standardised set of parameters for gyro-kinetic turbulence simulations and so may represent particularly strongly driven turbulence. This gives $w_{c} \sim 17 \rho_{i}$, which is $\sim 1 \mathrm{~cm}$ when using a gyro-radius of $2 \mathrm{~mm}$. This estimate is comparable to other physical mechanisms which determine the stability of the tearing mode.

\section{Conclusions}

Motivated by previous work on the nonlinear interaction of drift wave turbulence and magnetic islands formed by the growth of the tearing mode using both kinetic and fluid models, which have found that turbulence around and within a magnetic island can help to maintain a radial gradient in the trapped electron population.

It is shown via the use of a simple numerical model based on the drift kinetic equation in one spatial and one velocity space dimension, including the combined effect of turbulence and collisions, that the bootstrap current, which is responsible for the drive in the neoclassical tearing mode (NTM) and is driven by a gradient in the trapped electron population, may not be diminished within the magnetic island. The perturbation of the bootstrap current by the magnetic island is the drive for the NTM, and the consequence of the effect of turbulence and collisions on the trapped population is that the drive for the NTM is diminished.

The fact that the bootstrap current within the island is maintained modifies the threshold behaviour of the Neoclassical tearing mode. An estimate for the critical width of the magnetic island is comparable to those calculated for other contribution to the mode stability. This implies that the NTM may be more stable than previously thought.

\section{References}

[1] P. H. Rutherford, Phys. Fluids 161903 (1973)

[2] H. P. Furth, J. Killeen and M. N. Rosenbluth, Phys. Fluids, 6469 (1963)

[3] R. Carrera, R.D. Hazeltine and M. Kotschenreuther, Phys. Fluids, 29 899, (1986)

[4] Z. Chang, J. D. Callen, E. D. Fredrickson, R. V. Budny, C. C. Hegna, K. M. McGuire, M. C. Zarnstorff and TFTR group, Phys. Rev. Lett., 7423 (1995)

[5] H. R. Wilson, J. W. Connor, R. J. Hastie and C. C. Hegna, Phys. Plasmas, 3248 (1996)

[6] A. Smolyakov, P. Diamond, M. Yagi, K. Itoh and S. Itoh, Journ. Phys. Soc. Japan, 7611 (2007)

[7] R. Fitzpatrick, and F. L. Waelbroeck, Phys. Plasmas 16052502 (2009)

[8] A. Sen, R. Singh, D. Chandra, P. Kaw and D. Raju, Nucl. Fusion 49115012 (2009)

[9] A. G. Peeters, Plasma Phys. Control. Fusion 42 B231 (2000)

[10] E. Poli, A. G. Peeters, A. Bergmann, S. Günter, and S. D. Pinches, Plasma Phys. Control. Fusion $4571(2003)$

[11] W. A. Hornsby, A. G. Peeters, E. Poli, M. Siccinio, A. P. Snodin, F. J. Casson, Y. Camenen and G. Szepesi, Europhys. Lett., 9145001 (2010),

[12] W. A. Hornsby, A. G. Peeters, A. P. Snodin, F. J. Casson, Y. Camenen, G. Szepesi, M. Siccinio, and E. Poli, Phys. Plasmas 17, 092301 (2010) 
[13] E. Poli, A. Bottino, W. A. Hornsby, A. G. Peeters, T. Ribeiro, B. D. Scott, and M. Siccinio, Plasma Phys. Control. Fusion 52, 124021 (2010)

[14] E. Poli, A. Bottino and A. G. Peeters, Nucl. Fusion 49075010 (2009)

[15] A. G. Peeters, Y. Camenen, F. J. Casson, W. A. Hornsby, A. P. Snodin, D. Strintzi, and G. Szepesi, Comp. Phys. Comm. 180, 2649 (2009)

[16] A. Ishizawa, N. Nakajima, Nucl. Fusion 47, 1540-1551 (2007)

[17] Z. X. Wang, J. Q. Li, Y. Kishimoto and J. D. Dong, Phys. Plasmas 16060703 (2009)

[18] Z. X. Wang, J. Q. Li, J. D. Dong and Y. Kishimoto, Phys. Rev. Lett. 103015004 (2009)

[19] M. Muraglia, O. Agullo, S. Benkadda, X. Garbet, P. Beyer, and A. Sen, Phys. Rev. Lett., 103 $145001(2009)$

[20] H. R. Wilson and J. W. Connor, Plasma Phys. Control. Fusion 51115007 (2009)

[21] C. J. McDevitt, P. H. Diamond, Phys. Plamsas 13, 032302 (2006)

[22] R. Fitzpatrick, Phys. Plasmas 2, 825 (1995)

[23] M. A. Beer and G. W. Hammett, Phys. Plasmas 311 (1996)

[24] F. Y. Gang and P. H. Diamond, Phys. Fluids B 212 (1990)

[25] P. H. Rutherford, Phys. Fluids 132 (1970)

[26] P. J. Catto et.al., Phys. Fluids 302874 (1987)

[27] P. Helander Collisional Transport in Magnetised Plasmas Cambridge University Press

[28] R. E. Waltz, J. Candy, Phys. Plasmas 12, 072303 (2005)

\section{Acknowledgments}

This work was jointly funded by the EPSRC via the S\&I grant and UKAEA/Euratom via Mobility. This work used resources on the HECToR supercomputer that were provided by the Engineering and Physical Sciences Research Council [grant number $\mathrm{EP} / \mathrm{H} 002081 / 1]$. 